\title{
Une méthode orientée processus pour le pilotage par la performance des systèmes industriels
}

\author{
Matthieu Lauras - Jacques Lamothe - Hervé Pingaud \\ Centre Génie Industriel - Ecole des Mines d'Albi-Carmaux \\ Campus Jarlard, route de Teillet \\ 81000 Albi Cedex 09 - France \\ lauras@enstimac.fr, lamothe@enstimac.fr, pingaud@enstimac.fr
}

\begin{abstract}
RÉSUMÉ. Dans un contexte particulièrement instable et concurrentiel, les entreprises rationalisent leur organisation autour de processus connus, maîtrisés et adaptables. Parallèlement, elles développent une culture de mesure et d'évaluation de performance pour aider la prise de décision. A la croisée de ces deux évolutions se situe le pilotage de l'organisation par la mesure de performance de ses processus clés. Notre proposition est relative à ce type de pilotage, et basée sur un cadre de modélisation d'entreprise. La définition du système d'indicateurs de performance mobilise trois concepts principaux : les processus, les centres de décision et les déterminants de la performance. Le cadre de modélisation couvre ces concepts en préconisant l'usage des méthodes GRAI et SADT. Une architecture de pilotage est détaillée. Afin de structurer les informations produites au cœur même de cette architecture, une partition des indicateurs en trois classes est proposée : le « point de vue ambition »; le « point de vue réalité » et le « point de vue aisance ». Une application sur un exemple de chaîne logistique est présentée à titre d'illustration.
\end{abstract}

ABSTRACT. The main methods to help defining performance indicators give prominence to three concepts in order to manage the performance: processes, decision centres, performance determinants. The proposed approach is based on enterprise modelling tools (GRAI, SADT/IDEF0) and allows to integrate the best practices defined by these methods. Besides the indicators following through the results and determinants, three types of indicators are introduced to analyse the performance: the facility viewpoint measures the appropriateness of the resources available with the determinants of the activity. The ambition viewpoint evaluates with the determinants the feasibility of the aims assigned to the activity. The reality viewpoint measures the impact of determinants on the results of the activity. The implementation on the supply chain of an important health care company is presented.

MOTS-CLÉS : gestion de chaînes logistiques, évaluation de performance, système d'indicateurs de performance, déterminant de la performance, modélisation d'entreprise.

KEYWORDS: supply chain management, performance evaluation, performance indicators, performance determinant, enterprise modelling. 


\section{Introduction}

Un processus est une collection d'activités qui utilisent des ressources de manière à permettre au système de fonctionner, c'est à dire d'effectuer la transformation désirée d'éléments d'entrée en éléments de sortie [AFNOR, 2000]. Pour [Hammer et Champy, 1993], l'exécution est orientée vers la production d'un résultat représentant une valeur pour le client, une vision privilégiée caractérisant la chaîne de valeur. Le concept de valeur est toujours associé à une mesure de performance puisque, par définition, la valeur d'un résultat met en relation des qualités attractives qu'il possède avec l'énergie dépensée pour les obtenir.

L'objectif de toute organisation est de contrôler sa chaîne de valeur, ce qui oblige donc à mesurer la contribution de chaque fonction de l'entreprise à l'obtention du résultat. Une connaissance des activités qui composent ces fonctions permet d'assurer un pilotage du système, voire même la modification de sa manière de fonctionner par une démarche d'amélioration continue ciblée. Dans le management par processus [Morley, 2002], le système est appréhendé comme un ensemble de processus interconnectés, placés sous des responsabilités multiples dans l'organisation, et qui s'influencent mutuellement pendant leur exécution.

Selon [Amaratunga et Baldry, 2002], le management de la performance utilise des mesures pour induire des changements positifs dans l'organisation, et plus spécifiquement sur les processus. Le management de la performance s'applique classiquement en 4 phases [Smith et Goddard, 2002] : définir, mesurer, analyser et répondre. Les différents pilotes de processus ${ }^{1}$ ont donc à concevoir des systèmes de mesure de la performance qui leur permettent de mieux raisonner sur la performance de la partie du système dont ils ont la charge, et délivrer les préconisations de changement.

Parce que ces deux premières phases, définir et mesurer, conditionnent la qualité de ce type de management, elles doivent faire l'objet d'une attention particulière. Notre contribution se focalise d'abord sur cette partie du problème. [Gray, 1999] suggère de suivre 4 étapes pour définir et mesurer la performance : sélection des indicateurs, établissement du périmètre, détermination des objectifs (et de minima), définition d'une tolérance (raisonnable). Le choix d'un bon Indicateur de Performance (IP, i.e. une information qui décrit convenablement la performance du système étudié) est toujours délicat, nous ne nous étendrons pas sur cette vérité bien connue des praticiens. L'établissement du périmètre est une question de plus en plus délicate dans le contexte actuel. En effet, si l'indicateur de performance agrége des données provenant de plusieurs fonctions différentes, et souvent de plusieurs responsables, au sein d'une même organisation, le problème est déjà subtile. Mais si

${ }^{1}$ Un pilote de processus est entendu au sens de l'entité ou la personne qui a la responsabilité du processus en question. 
l'IP couvre plusieurs organisations, la mise en commun d'informations, et la production d'IP pertinents est encore plus délicate. Un contexte de systèmes à caractère distribué implique des cadres de représentation du fonctionnement d 'ensemble. Les chaînes logistiques ou les groupements d'entreprises [Beamon, 1999] sont des cas typiques de cette situation. Plusieurs auteurs [Lee, 2000][AlMudimigh et al., 2004] ont déjà soutenu cette idée que le management de la performance de telles organisations requiert nécessairement une connaissance des processus de gestion, incluant une prise en compte de la fonction de coordination. Ils jugent notamment que, dans de telles situations, l'utilisation uniquement de mesures locales n'est pas suffisante. [Folan et Browne, 2005] avancent même l'idée que le développement de systèmes de mesure de la performance disparates peut conduire à des prises de décision incompatibles. Se pose ainsi le problème du management de la performance tout autant des processus internes que des processus plus collectifs.

Le poids de la coordination dans le pilotage des entreprises aujourd'hui nous a conduit à placer les modèles d'entreprise au cœur de la démarche de conception du système de mesure de performance. Un tel choix induit une approche normative du problème, puisqu'il fixe des contraintes de conception pour chaque élément d'organisation, qui rentrera dans une architecture déterminée avec le même cadre de modélisation. Mais, en échange, il facilite l'acte de conception ou de reconception à l'échelle de l'organisation, ce qui constitue un facteur clé de succès dans le paysage industriel actuel.

La phase d'analyse de la performance prolonge la définition et la mesure des IP. Afin de la réaliser dans les meilleures conditions, nous avons cherché à établir très en amont des rapports fructueux entre la mesure et la décision en travaillant sur le sens des axes d'analyse de la performance. En développant des points de vue de la performance autour de cadres de décision, et à caractère couvrant par rapport aux processus, nous aurons la possibilité de construire des tableaux de bord structurés.

La partie 2 présente une analyse bibliographique sur la performance et son évaluation. La partie 3 étudie la mise en pratique des méthodes d'évaluation de performance. La partie 4 présente notre proposition. La partie 5 formalise notre méthode d'aide à la définition de systèmes d'indicateurs de performance pour le pilotage de processus. La partie 6 décrit le déploiement de cette proposition sur la chaîne logistique d'une entreprise pharmaceutique et cosmétique.

\section{Analyse bibliographique de l'évaluation de performance a posteriori}

L'évaluation de performance est utilisée, soit pour concevoir un nouveau système (ou modifier un système existant), soit pour piloter un système existant. On parle alors respectivement de démarches d'évaluation a priori ou a posteriori [Frein, 1998][Tahon et Frein, 1999] s'appuyant sur des indicateurs de performance ex ante (aider la prise de décision à venir) ou ex post (évaluer la qualité des décisions passées) [Giard, 2003]. 
[Burlat et Boucher, 2003] précisent qu'une démarche d'évaluation de performance, qu'elle soit a priori ou a posteriori, a besoin d'un modèle d'interprétation du système réel pour décrire les liens de causalité entre des actions sur le système et des performances en sortie. C'est ce lien qui nous projette dans le domaine du pilotage de système. Car, comme en commande classique, trois notions structurent la notion de pilotage par la performance [Bitton, 1990][Berrah et Haurat, 1997] [Berrah et al., 2000] :

- l'objectif représente l'état espéré du système piloté,

- la mesure rapporte l'état réel constaté de ce même système,

- la variable de décision constitue un levier sur lequel on peut agir dans le but de réduire l'écart entre l'objectif affiché et son estimation à partir de la mesure.

La déclinaison sur un système distribué de taille réelle pose le problème du nombre de mesures, d'objectifs et d'actions possibles, autant que de leurs interactions. Il est souvent difficile d'envisager une démarche d'évaluation de performance a priori ${ }^{2}$ parce que les liens entre mesures, actions et trajectoire suivie sont trop complexes, la connaissance est limitante. Très souvent, le système ne peut être décrit formellement à un niveau tel qu'il puisse servir de base pour des calculs prédictifs. L'alternative, l'évaluation de performance a posteriori, apparaît en pratique moins exigeante en termes de représentation par des modèles quantitatifs. Elle cherche d'abord à établir une simple mesure de la performance du système étudié, puis à exploiter ces données pour piloter la performance en jouant sur les liens aux variables d'action influentes, et cela quelque soit le degré de complexité du système [Burlat et al., 2003]. Cette approche du pilotage repose en quelque sorte sur une considération des trois notions de commande énoncées plus haut en deux parties :

- le contrôle, c'est à dire la considération des objectifs et la définition de la trajectoire,

- la régulation qui est le maintien du système dans les limites du cadre prédéfini par la mesure et les actions.

Plusieurs auteurs ont proposé des revues de la littérature sur les thèmes de la mesure de la performance, de l'évaluation de performance et/ou du management de la performance [Chow et al., 1994][Neely et al., 1995][Beamon, 1999] [Folan et Browne, 2005]. Ils évoquent en particulier la conception d'un système d'indicateurs de performance dans le contexte d'une organisation productive. Qu'ils soient inscrits dans le courant du contrôle de gestion ou celui du management des opérations, ces auteurs abordent principalement les questions des rapports entre opérations et

${ }^{2}$ L'évaluation de performance a priori porte toujours, par définition (évaluation de l'impact d'une variable d'action sur le comportement du système), sur des systèmes « commandables ». 
performances, et du lien entre stratégie et performance [Lohman et al., 2004]. Nous faisons un rappel de certains de ces travaux avec une trame légèrement différente. La performance est étudiée par rapport à trois sujets : sa mesure sur les processus opérationnels, sa prise en compte dans les processus décisionnels, et enfin les axes d'analyse qui constituent une interface entre mesure et action.

\subsection{Relations entre performance et processus opérationnels}

\subsubsection{Activity Based Costing et Activity Based Management}

Développées pour le contrôle de gestion de l'entreprise, l'Activity Based Costing $(A B C)$ et l'Activity Based Management (ABM) délivrent des informations pertinentes sur les coûts et sur les marges [Ravignon et al., 1998][Cokins, 2001]. Les coûts des produits sont établis par des informations tirées de la consommation effective de moyens par les activités. Dans ce type de démarche, il n'y a plus de distinction entre les coûts indirects et directs car toutes les charges sont supposées être ventilées sur des activités. La mise en œuvre de démarche $A B C$ se décline en cinq temps :

- Définition d'une cartographie des processus,

- affectation des charges et temps de travail aux différentes activités,

- définition d'un ensemble d'indicateurs mesurant la production de l'activité,

- identification des quantités de ressources consommées par produit et activité,

- détermination du coût de revient du produit, en coût total et en coût unitaire, détaillé par activités.

La mesure des coûts, si elle est un but ultime, impose une structuration par le processus. La performance est suivie par des indicateurs placés sur ces processus qui sont en ligne avec une perception voulue de la réalité du fonctionnement.

L' $A B M$ prolonge la démarche $A B C$ en proposant une gestion par activités au moyen de tableaux de bord où les mesures sont reportées par catégorie : les progrès enregistrés suite à la mise en oeuvre d'actions décidées conformément à la stratégie, la valeur perçue par le client, les performances des concurrents, la démarche qualité et l'amélioration des performances.

\subsubsection{SCOR}

Le modèle SCOR (Supply Chain Operation Reference) permet d'avoir une vision sur l'ensemble d'une chaîne logistique en facilitant la représentation des flux physiques, informationnels et financiers. C'est un référentiel utilisé pour la comparaison des performances logistiques entre entreprises d'un même secteur. Pour ce faire, SCOR propose des outils d'analyse, et des outils d'évaluation [Supply Chain Council, 2004] classés en quatre niveaux de granularité. 
Le niveau 1 permet de modéliser le périmètre d'une chaîne logistique avec une vision très macroscopique selon une base de fonctions principales appartenant à la chaîne de valeur (approvisionner, faire, délivrer, planifier et retourner),. Le niveau 2 détaille chacune de ces fonctions élémentaires grâce à 26 catégories de processus prédéfinis. Le niveau 3 prolonge le détail de chacun des processus définis au niveau 2. Le niveau 4 est celui des activités élémentaires, spécifiques à chaque entreprise. Dans l'optique d'une analyse qui succéderait à l'évaluation de la performance du système par le modèle SCOR, trois catégories d'indicateurs sont proposées et affinées sur les trois premiers niveaux [Bolstorff, 2002] :

- $\quad$ évaluation pour les clients : qualité de service, flexibilité, vélocité,

- évaluation des processus internes : coûts et productivité,

- évaluation pour les actionnaires : rentabilité, retour sur investissement et dividendes.

Bien que ces indicateurs de performance soient parfaitement décrits, le déploiement de ce système d'évaluation au sein d'une chaîne logistique reste difficile. En effet, si la décomposition des processus à un niveau macroscopique est suffisamment générale pour être appliquée à n'importe quel cas d'étude, les niveaux plus détaillés (niveau 2 et surtout niveau 3 ) ont des contenus qu'il devient difficile d'identifier sur un système existant. L'organisation considérée est supposée décloisonnée, et entrant parfaitement dans le «moule» proposé par le modèle SCOR, ce qui n'est pas toujours le cas.

Le modèle SCOR ne fait pas référence à des moyens à mettre en œuvre, après analyse, pour assurer un niveau de performance escompté. Il est donc, à l'usage, assez difficile - voire aléatoire dans certains cas - de définir sur cette seule base, les actions correctrices attendues en cas de défaillance.

\subsection{Relations entre performance et processus décisionnels}

\subsubsection{Le Balanced Score Card}

La méthode du «Balanced Score Card» (BSC) permet de définir des cibles stratégiques et de les décliner en buts opérationnels concrets pour une organisation. Cette approche complète naturellement le cadre de gestion par la performance avec une contribution originale sur la définition des objectifs, et les moyens de les atteindre. L'approche de Kaplan et Norton [Kaplan et Norton, 1996] part du postulat que la stratégie est définie comme la vision, dictée par la direction, de ce qui doit être fait sur les processus internes pour atteindre une cible privilégiée. L'évaluation de la performance est, là encore, considérée comme le résultat de la mise en œuvre des processus [Kaplan et Norton, 2001]. La performance est induite par l'utilisation pertinente de variables, appelées déterminants de la performance, pour obtenir les résultats voulus avec des moyens adaptés et selon des consignes explicites. Les déterminants de la performance sont définis comme l'ensemble des variables de 
décision relatives aux clients, aux processus internes et à l'apprentissage organisationnel qui s'appuient sur une traduction claire et rigoureuse de la stratégie en objectifs, et qui influent sur la performance de l'organisation.

Nous pensons qu'un des apports fondamentaux du BSC est d'introduire ces déterminants comme le moyen de fermer la boucle de pilotage du système.

On sait qu'une telle mise en relation entre le terrain et la stratégie est ambitieuse, et qu'un succès passe par une attention particulière de l'organisation sur deux notions clés :

- la cohérence qui garantit l'adéquation avec la stratégie au niveau de chaque activité et de chaque ressource (liaison verticale) ;

- la convergence qui mobilise tous les acteurs pour établir des liaisons entre les centres de profit, les services communs et les individus (liaison horizontale).

Ces deux notions clés sont très proches de celle de coordination et d'intégration dans le pilotage des systèmes de production.

Quatre axes d'analyse sont proposés pour raisonner sur la performance d'un système : satisfaction du client, résultat financier (rentabilité ou CA en fonction de la stratégie), fonctionnement interne, apprentissage et développement. Sur chacun de ces axes, quatre types de processus clés doivent être évalués: processus d'innovation, processus de gestion du client, processus opérationnel, et processus réglementaires ou écologiques.

Il est clair que le BSC qui porte l'évaluation au niveau stratégique du système entreprise doit considérer d'autres types de processus que ceux qui sont en ligne avec la production au sens strict, et tenir compte des capacités à renouveler le portefeuille de produits ou encore les relations au reste de l'écosystème, par exemple. Une mauvaise performance sur ces processus est une cause possible de défaillance sur les horizons de temps correspondant à une évaluation de la stratégie.

\subsubsection{ECOGRAI}

En modélisation d'entreprise, la méthode GRAI est une des méthodes les plus abouties sur la prise en compte de la décision. Une variable de décision est un facteur, ou un ensemble de facteurs, sur lequel on agit, afin de faire évoluer tout ou partie d'un processus ou d'un système. C'est la variable sur laquelle on doit agir pour améliorer la performance [Bitton, 1990]. Elle représente un moyen d'action, une commande mise à la disposition du décideur pour modifier le comportement du système piloté.

L'approche ECOGRAI développe l'idée que la performance doit être associée aux variables de décision (ou déterminants de la performance). Elle aide à la définition d'un système d'indicateurs de performance pertinent et cohérent avec les variables de décision. 
ECOGRAI est conçue pour assurer cette cohérence au travers d'une méthodologie d'élaboration et d'utilisation appropriée des indicateurs [Ducq et al., 2001]. La méthode repose sur une démarche en cinq temps [Bitton, 1990][Ducq et al., 2003] :

- établir le modèle de la structure de pilotage du système étudié à l'aide d'une grille GRAI [Doumeingts, 1984], et identifier les centres de décision dans lesquels implanter des indicateurs;

- identifier les objectifs qui sont attachés à chaque centre de décision ;

- trouver les leviers à partir desquels les décideurs peuvent agir pour atteindre leurs objectifs (variables de décision) ;

- proposer des indicateurs de performance. Une analyse de cohérence, au moyen d'une table de correspondance, évalue l'adéquation de chaque indicateur avec les variables de décision et les objectifs ;

- développer, implanter et maintenir le système d'information des indicateurs.

Si le cadre très formel d'ECOGRAI est un moyen puissant pour tenter d'influer sur la performance, il n'offre pas nativement de directive pour expliquer la performance. La relation causale à la décision pour déterminer les origines d'une déficience reste un savoir faire de l'évaluateur. Le fait d'implanter les indicateurs sur les centres de décision masque en partie les processus opérationnels dont l'importance est mise en avant par des méthodes déjà présentées $(B S C, A B C / A B M)$.

Il y a une dépendance entre déterminants de la performance et variables de décision inscrite dans la définition du déterminant de la performance fournie par BSC. La grille GRAI impose une partition des variables de décision suivant une décomposition fonctionnelle, qui constitue un point d'entrée possible de la représentation des processus, et une décomposition temporelle. A l'intersection d'une fonction et d'un horizon de temps se situe un centre de décision, objet particulièrement structurant pour le pilotage.

Il y a une sorte de complémentarité assez naturelle entre ces deux démarches de pilotage par la performance (BSC et ECOGRAI) qui ne considèrent pas le processus de la même façon, complémentarité que nous chercherons à exploiter.

\subsection{Relations entre performance et axes d'analyse}

La pertinence, l'efficience et l'efficacité et (PEE) sont des qualifiants, définis par des ratios simples établis par permutation circulaire autour du triplet objectifs/moyens/résultats [Jacot, 1990]. Ce sont des concepts qui se conjuguent très bien avec la notion d'activité ou de processus. [Burlat, 2002] reprend les définitions proposées par [Bescos et al., 1995] et présente : 
- la pertinence comme «l'adéquation entre moyens mis en œuvre et objectifs. Son évaluation renvoie au réalisme des moyens alloués par rapport aux objectifs visés »;

- l'efficacité comme «la caractérisation de l'écart entre les objectifs à atteindre et les résultats obtenus. Elle mesure l'aptitude du système de pilotage à tenir les objectifs qui lui ont été assignés »;

- l'efficience comme « la mesure du rendement du système par comparaison entre les moyens mis en œuvre et les résultats obtenus ».

Ces concepts vont, pour nous, devenir des axes d'analyse sur les activités et les processus. Ainsi, le processus portera non seulement ses propres capteurs de performance, mais il sera aussi accompagné de règles simples de classification des indicateurs pour faciliter l'analyse. De par la présence des objectifs et des moyens, le rapport à la décision n'est pas éliminé du raisonnement. Une approche de ce type reste générique, et prend le contre pied des définitions plus spécifiques des axes d'analyse comme le propose le BSC, par exemple.

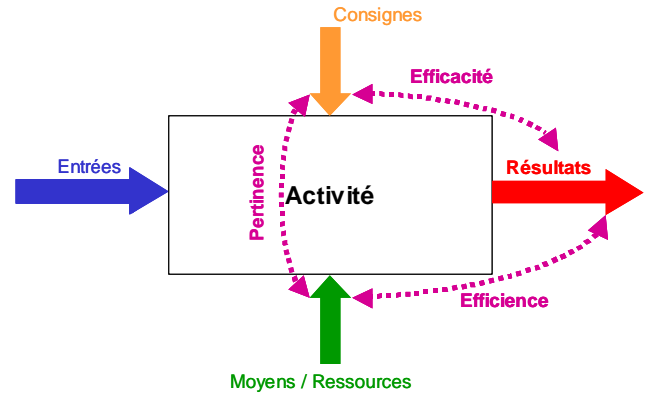

Figure 1. Triptyque Pertinence, Efficacité, Efficience (d'après [Jacot, 1990], [Bescos et al., 1995][Burlat, 2002]) .

\subsection{Synthèse de l'analyse bibliographique}

Une des vocations de l'indicateur de performance [Giard, 2003] est d'apporter un éclairage aux décideurs. Lorsqu'une connaissance explicite des processus est disponible, toute la démarche de management de la performance devrait s'articuler autour d'elle, depuis la définition des indicateurs jusqu'à l'action.

L'imbrication entre décision et activité est inscrite dans la typologie classique qui distingue les processus de pilotage des processus opérationnels [Debauche et Mégard, 2004] :

- les processus de pilotage fixent les finalités (consignes) et les moyens (ressources) de tous les autres processus. Ce sont eux qui caractérisent la prise de décision et la véhiculent vers les autres processus ; 
- les processus opérationnels sont des enchaînements d'activités qui utilisent des ressources, transforment des entrées en sorties en fonction de consignes préétablies par les processus de pilotage. Ces enchaînements doivent être structurés de façon à produire un résultat constituant de la valeur pour le client,

Le premier type fixe les enjeux de la performance par les consignes, et la façon de la réaliser à travers les moyens. Alors que le second type réalise la performance en fonction de ces consignes et moyens qui lui sont assignés.

Il est absolument nécessaire de conserver une vision claire des relations entre les deux types de processus dans une optique de pilotage par la performance. Les apports des travaux antécédents à notre étude sont schématisés sur la figure 1. La performance est :

- dictée par différents processus de pilotage, que nous appréhendons par les centres de décision présents dans l'organisation (ECOGRAI, BSC),

- induite par les déterminants de la performance, qui sont des variables de décision (BSC) qui influencent le déroulement des activités opérationnelles,

- déterminée par les conditions de réalisation des activités des processus opérationnels (ABC / ABM, SCOR).

Dans le cadre d'une démarche à posteriori. la capacité d'analyse se fonde sur des informations relatives aux trois composantes complémentaires que nous venons de lister. Nos axes d'analyse expriment les rapports entre objectifs, moyens et résultats, ils servent de base au raisonnement qui fermera la boucle entre processus opérationnels et processus de pilotage.

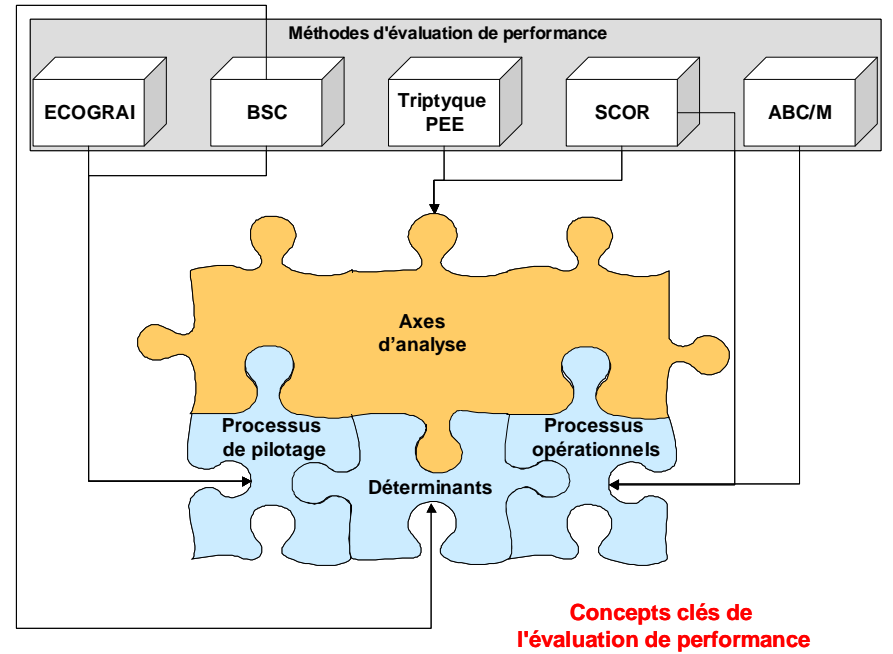

Figure 2. Concepts clés de l’évaluation de performance. 
Notre problème consiste à composer une méthodologie complète et instrumentée autour de ces principes.

\section{La modélisation d'entreprise pour l'instrumentation de l'évaluation de performance}

Un système industriel est, par nature, un système complexe. La modélisation d'entreprise fournit des solutions pour s'attaquer à cette complexité. La norme ISO 19439 définit un cadre de modélisation d'entreprise qui repose sur trois dimensions d'analyse:

- la dimension "généricité » définit le niveau de détail du modèle d'entreprise : générique ; partiel qui propose des structures prédéfinies et réutilisables pour un domaine d'application donné (SCOR par exemple) ; particulier à une entreprise. Notre travail concerne un système existant. Nous cherchons à proposer un système d'indicateurs de performance spécifique à ce dernier. Cette dimension de généricité n'a pas d'apport particulier.

- la dimension «abstraction » spécifie l'étape étudiée du cycle de vie du système (Identification, Conceptualisation, Spécification des besoins, Conception, Implémentation, Réalisation des opérations et Démantèlement). Travaillant sur un système en exploitation, notre but est de spécifier les besoins en termes d'évaluation de performance, de concevoir le système d'indicateurs de performance et de proposer une démarche d'implantation du tableau de bord ainsi constitué. Nous avons donc à la parcourir dans son intégralité. Par la suite, nous nous attachons à décrire les phases de spécification et de conception du système.

- la dimension «vue» précise la nature des objets impliqués dans une représentation du système : la vue ressource présente les moyens utiles pour mettre en œuvre les fonctions du système ; la vue informationnelle décrit les objets du système et leurs relations ; la vue fonctionnelle représente le comportement du système en particulier les processus, activités et opérations ; la vue organisationnelle présente la distribution des acteurs et des rôles. Pour être complet, il faut ajouter une vue qui apparaîtra dans une évolution prochaine de la norme : la vue décisionnelle.

Les centres de décision seront le point d'entrée de la description des processus de pilotage qui forment la vue décisionnelle. Les processus opérationnels seront représentés dans la vue fonctionnelle du système.

La nature multi-acteurs et multi-sites des systèmes logistiques nécessite de situer les responsabilités associées aux activités qui composent ces systèmes dans la vue organisationnelle. Les besoins étant assez restreints (identification des responsabilités et de la coordination des tâches), nous avons opté sur ce point particulier pour des modèles simples : organigramme et identification graphique des acteurs associés aux activités ou aux décisions. 
Le cadre de modélisation préconisé est très proche de celui de GIM [Doumeingts et Vallespir, 2004] :

- utilisation d'actigrammes pour la vue fonctionnelle : SADT / IDEF0 ${ }^{3}$. Ce sont des représentations très usitées et que l'on retrouve dans $\mathrm{ABC} / \mathrm{ABM}$ ou dans SCOR ;

- représentation de la vue décisionnelle à l'aide de la grille fonctionnelle GRAI

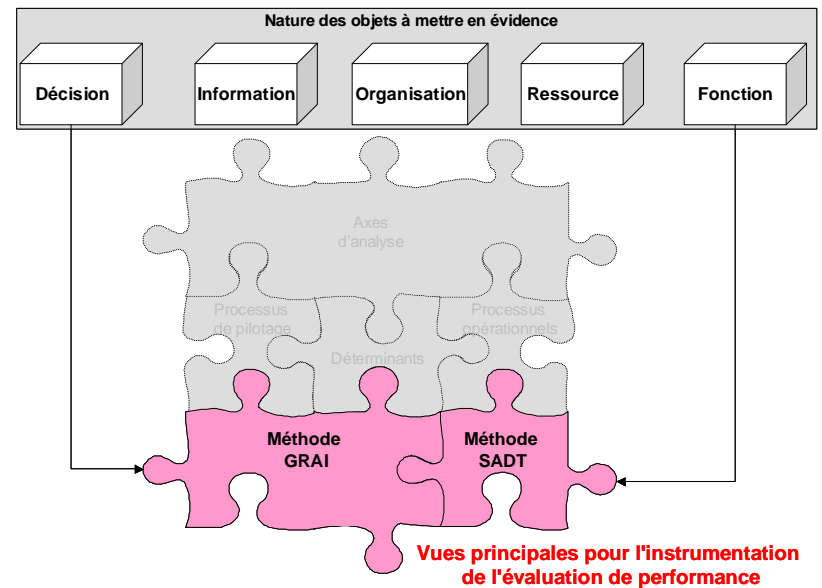

Figure 3. Modélisation associée à l'architecture de notre système de définition d'IP

\footnotetext{
${ }^{3}$ SADT selon [Lissandre, 1990] : Une activité consomme des entrées pour produire des sorties à partir de directives de contrôle (informations) en s'appuyant sur les potentialités des mécanismes (ressources).

${ }^{4}$ GRAI selon [Doumeingts, 1984][Roboam, 1993] : un centre de décision est le croisement d'une fonction et d'un couple "horizon de planification/ période de replanification". Les centres de décision sont positionnés au sein d'une grille GRAI (cf. Figure 8 de l'application). Chaque cadre de décision précise les performances attendues de cette décision (les objectifs), les variables de décision, les contraintes sur ces variables et des critères de choix parmi les actions possibles. La grille GRAI permet de différencier les liaisons dites décisionnelles (double flèche) des liaisons dites informationnelles (simple flèche).
} 


\section{Définition du système d'indicateurs de performance}

\subsection{Principes généraux de l'architecture de pilotage}

Selon plusieurs auteurs [Berrah, 2002][Folan et Browne, 2005], le pilotage peut se décomposer en plusieurs niveaux de portée différente :

- le pilotage opérationnel qui correspond principalement à la régulation d'un processus du système physique par rapport à une consigne préétablie.

- Le pilotage tactique qui consiste à adapter les ressources existantes au vu de l'état constaté.

- Le pilotage stratégique qui a pour rôle de veiller à corriger les éventuelles dérives au regard des objectifs stratégiques.

Le principe de l'articulation entre ces différents niveaux a été abordé dans les travaux de [Lohman, 1999], [Lohman et al., 2004] et [Folan et Browne, 2005]. Ils proposent une schématisation par une double boucle de pilotage que nous avons reporté sur la figure 3. Les pilotages stratégique et tactique sont fusionnés.

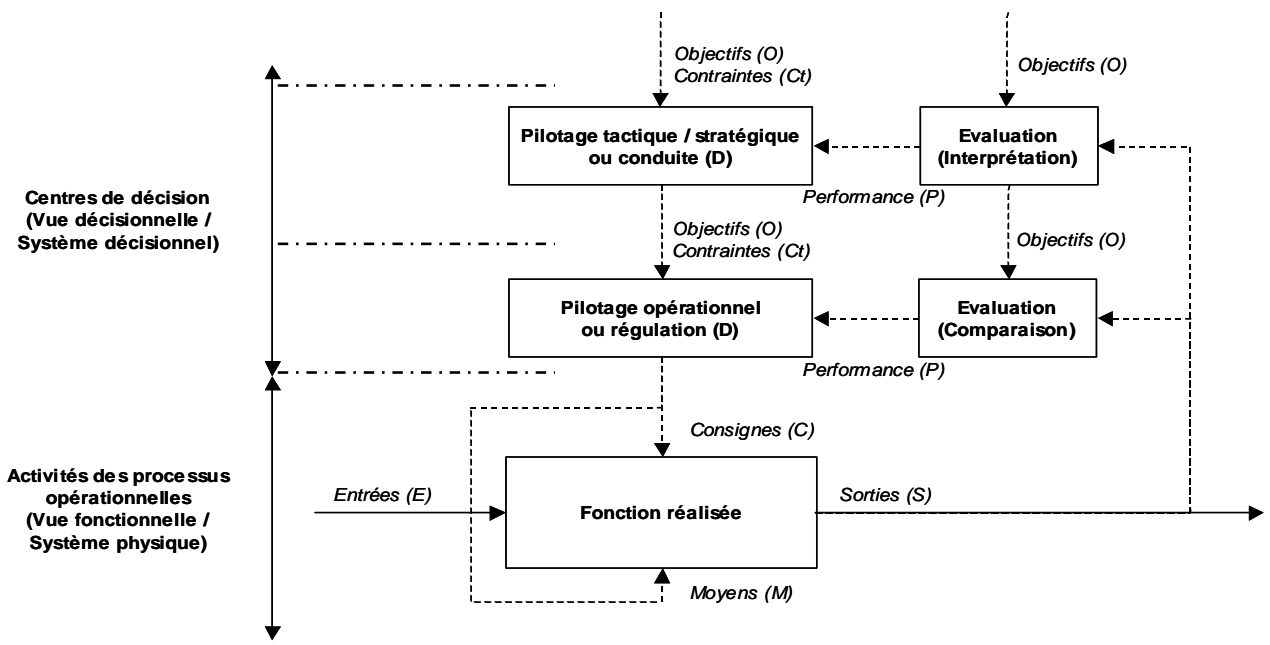

Figure 4. Principe d'articulation entre les vues fonctionnelle et décisionnelle : Boucles de pilotage (d'après [Lohman, 1999] et [Lohman et al., 2004]).

Un centre de décision correspond à un ensemble d'activités contribuant à une même fonction, et ayant même horizon de planification et période de remise en cause. Ces activités doivent être exécutées pour atteindre des objectifs donnés dans un cadre de décision (contraintes) [Doumeingts et Vallespir, 2004]. L'objet du centre de décision est de produire des consignes et d'affecter des moyens pour tout 
ou partie d'un processus opérationnel lui étant rattaché. A cette fin, le centre de décision détermine les valeurs des variables de décision à partir des informations externes et des résultats de l'évaluation de performance, pour satisfaire au mieux les objectifs et respecter les contraintes. Le centre de décision est une forme de représentation très condensée de l'activité de pilotage. En cela, l'assimilation d'un centre de décision avec une action de pilotage telle que décrite dans la figure 3 semble tout à fait légitime.

Au niveau du pilotage opérationnel, on retrouvera la boucle classique de pilotage fondée sur le principe de la réaction ([Mélèse, 1990] par exemple). L'évaluation de performance $(\mathrm{P})$ consistera à comparer les mesures effectuées sur les sorties de l'activité $(\mathrm{S})$ du processus opérationnel avec les objectifs $(\mathrm{O})$ considérés comme des consignes (cf. Figure 4). Si une anomalie est constatée entre la valeur de l'indicateur et l'objectif recherché, alors une ou plusieurs décisions (D1) sont prises pour corriger le comportement dynamique du système afin de résoudre le problème. Ainsi, un ensemble D1 de décision définira des consignes (C) et/ou des moyens (M) pour l'activité concernée.

La deuxième boucle concerne les centres de décision de plus haut niveau (tactique et stratégique). Les constantes de temps sont plus élevées. Ces niveaux de pilotage travaillent généralement sur une prédiction du comportement à travers un programme. Ce programme est un ensemble de consignes et d'affectation de ressources pour un intervalle de temps fixé. Les auteurs indiquent qu'elle permet d'évaluer et surtout d'adapter la boucle de pilotage de niveau inférieur en précisant le cadre de décision (objectifs $(\mathrm{O})$ et contraintes $(\mathrm{Ct})$ ) à considérer. Le principe de l'horizon glissant permet de gérer la cohérence entre les différents niveaux.

A partir de ce schéma, il est possible de déduire les relations de causalité qui lient les différentes variables intervenant dans le pilotage du système, sur un exemple simple. Pour ce faire, nous utilisons les diagrammes de causalité ${ }^{5}$ issus de la dynamique des systèmes proposée par [Forrester, 1968]. La figure 4 montre un tel diagramme en analogie avec le figure 3, c'est à dire pour deux centres de décision superposés l'un à l'autre, et connectés à une seule fonction au niveau opérationnel.

\footnotetext{
${ }^{5}$ Les diagrammes de causalité sont un moyen simple pour représenter la structure d'un système, à savoir les variables caractéristiques et les liens de causalité. Les variables du système interagissent les unes par rapport aux autres dans des relations de cause à effet. La relation de cause à effet entre une variable x produisant un effet sur une variable y est représentée par une flèche pointant de $\mathrm{x}$ vers $\mathrm{y}$. Ces liens peuvent être de polarité positive ou négative (signe + ou - ). Les polarités n'ont pas été retenues dans notre diagramme.
} 
Centres de décision (Vue décisionnelle / Système décisionnel)

Activités des processus opérationnelles (Vue fonctionnelle / Système physique)

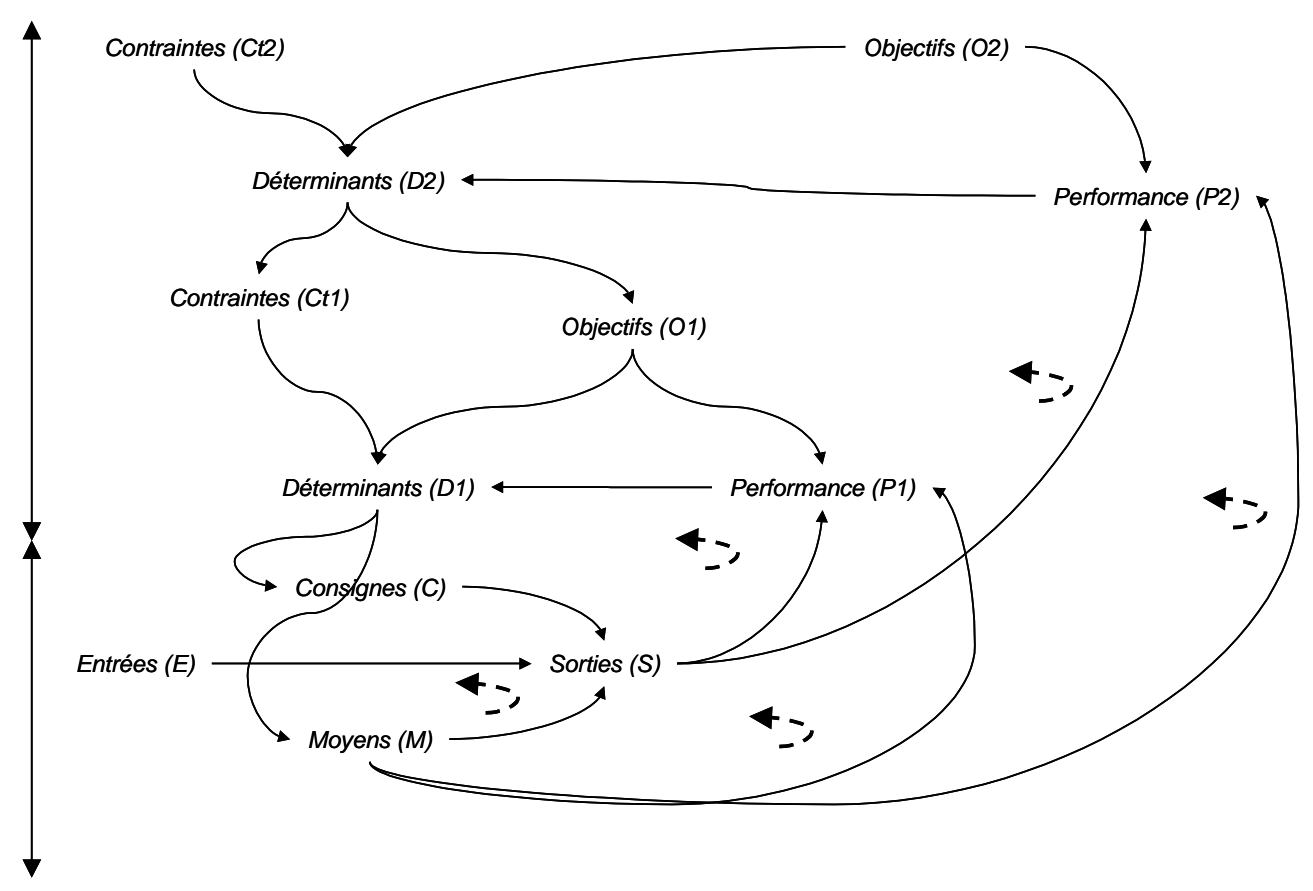

Figure 5. Diagramme de causalité du système de pilotage.

Les décisions prises au niveau du pilotage opérationnel (déterminant D1) ont un effet sur le dimensionnement des moyens et des consignes assignés à l'activité de transformation. Ces moyens et consignes impactent directement les sorties, qui sont également, et naturellement, influencées par les entrées de l'activité.

Par rapport à l'objectif $\mathrm{O} 1$, une variable de performance (P1) traduit l'effet de la valeur de la variable sortie (S) Plus les sorties seront conformes aux objectifs, plus la performance sera bonne, au sens de l'efficacité du système. Mais, la performance se juge aussi à l'aune des moyens alloués pour réaliser l'activité et de l'utilisation qui en est faite, c'est l'efficience du système. Nous avons donc tissé une relation entre la variable moyen $(\mathrm{M})$ et la performance $(\mathrm{P} 1)$.

Une logique tout à fait similaire nous permet de renseigner les relations de cause à effet sur les niveaux supérieurs de décision. Ainsi, il apparaît que la performance (P2) relative à l'activité pilotée depuis ce centre de décision est la comparaison de l'ensemble des sorties (S) avec les objectifs (O2), sous conditions des moyens (M).

La prise de décision qui génère des valeurs de D1 (resp. D2) à partir des performances enregistrées P1 (resp. P2) et des objectifs affichés O1 (resp. O2), est réalisée en tenant compte de Contraintes Ct1 (resp.Ct2). 


\subsection{Définition préliminaire des axes d'analyse de la performance}

Gardant le principe de comparaison présent dans le triptyque pertinence efficacité - efficience (PEE), préconisé par [Jacot, 1990][Bescos et al., 1995] et [Burlat et Boucher, 2003] et dont nous avons déjà commenté le bien fondé pour structurer la phase d'analyse, nous introduisons trois nouvelles catégories d'indicateurs de performance, nommées "points de vue» qui ont la propriété d'inclure les déterminants de performance dans la comparaison :

- le point de vue «ambition» évalue l'adéquation des consignes avec les déterminants de la performance. Il s'agit, dans ce cas, de juger de la faisabilité des consignes assignées à l'activité. Les consignes peuvent effectivement être en décalage par rapport aux types d'actions conduites au sein de cette activité. Cette information doit permettre de détecter des définitions par les déterminants de consignes farfelues ou inadaptées à la mission confiée. On mesure finalement ici la relation cause-effet des déterminants $\mathrm{D}$ sur les consignes $\mathrm{C}$.

- le point de vue «réalité » évalue l'adéquation des résultats de l'activité avec les déterminants. Il s'agit de préciser dans quelle mesure la mise en œuvre des déterminants de la performance de l'activité a bien produit les résultats attendus. Cet élément doit permettre de valider ou mettre en question les choix établis en termes de leviers d'action ou de décision. On mesure finalement ici la relation cause-effet des déterminants $\mathrm{D}$ sur les résultats $\mathrm{S}$.

- le point de vue «aisance» évalue l'adéquation des moyens avec les déterminants de la performance. Il s'agit ici de jauger dans quelle mesure les déterminants définissent des moyens et des ressources permettant de supporter l'exécution de l'activité. Cette donnée doit permettre de détecter des moyens insuffisants ou des ressources surdimensionnées. On mesure finalement ici la relation cause-effet des déterminants D sur les moyens $\mathrm{M}$.

Il faut insister ici sur le fait que l'introduction de ces points de vue constitue bien une nouveauté par rapport au triptyque PEE. Si ce dernier permet l'analyse de l'impact sur la performance des éléments de soutien à la réalisation d'une activité (ressources, consignes) d'une part, et des éléments produits par l'activité (sorties) d'autre part, en aucun cas, ces axes d'analyse n'offrent la possibilité de déterminer l'impact sur la performance des facteurs d'influence que constituent les déterminants. C'est tout l'enjeu de notre proposition de substituer ces nouveaux points de vue au triptyque PEE.

Ces points de vue seront mis en œuvre de manière systématique, à partir de la connaissance des processus opérationnels, et de l'exploitation des relations aux déterminants de la performance. La Figure suivante situe ces éléments sur une seule activité symbolique avec une représentation sous forme d'actigramme. Sur cette figure, les déterminants de performance $\mathrm{D}$ ont été substitués au nom de l'activité, ce qui permet de définir les liens entre les informations composant un point de vue. 


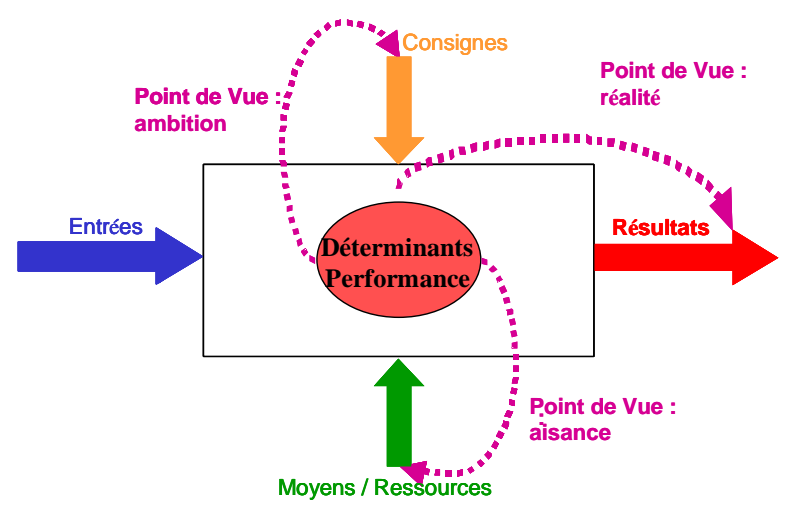

Figure 6. Les points de vue proposés pour l'analyse des performances.

L'approche que nous exposons présente un lien très mécaniste entre les déterminants et la performance. La réalité industrielle est plus complexe et, des approches plus cognitives invitent à nuancer nos propos. Le problème porte, au-delà de l'exactitude de la mesure du résultat, sur l'incidence de mesurer le comportement des acteurs: qui dit déterminant de la performance, dit décision et donc responsabilité. La définition, et a fortiori la mise en œuvre, de systèmes d'indicateurs de performance ne peut faire abstraction des valeurs comportementales de chacun et de leur conséquence sur la performance [Burlaud, 1999]. Ne pouvant développer cette thèse dans cet article, nous invitons le lecteur à se référer aux travaux de [Bourguignon, 1997].

\subsection{De l'analyse de la performance au pilotage}

L'approche que nous proposons, par contre, permet d'envisager, à partir des trois points de vue, un lien direct entre la mesure de la performance et des actions de progrès, correctives ou curatives ciblées (car portant directement sur les possibilités d'action du centre de décision). Il s'agit d'entrer dans une démarche de pilotage par la performance. La notion de pilotage par la performance (ou de pilotage de la performance) est associée aux systèmes complexes, difficilement maîtrisables [Burlat et al., 2003]. Elle caractérise les processus qui permettent de maîtriser et guider le fonctionnement et l'évolution d'un système [Morley, 2002].

Cette approche du pilotage repose sur deux activités fondamentales : le contrôle (considération des objectifs) et la régulation (maintien du système dans les limites du cadre prédéfini). 


\subsection{Développement des modèles}

Des conditions de mise en oeuvre de notre approche doivent être respectées, il faut s'assurer que :

- les déterminants de la performance peuvent être identifiés dans les processus de pilotage. Ce sont les leviers de commande des centres de décision,

- il est possible de définir les relations entre les activités d'un processus de pilotage et les activités des processus opérationnels: un centre de décision associe ses déterminants de la performance aux activités opérationnelles qu'il pilote et dont il est responsable. Par exemple, le processus de pilotage « réaliser le Plan Industriel et Commercial » pourra être associé au processus opérationnel «allouer stocks familles produits», « fabriquer les familles de produits » et « approvisionner les familles de composants ».

Nous proposons de modéliser les processus de pilotage par une ou plusieurs grilles GRAI (grilles fonctionnelles). Horizontalement, apparaissent dans une grille GRAI des centres de décision raisonnant sur un même horizon de temps et remettant en cause leurs décisions à la même fréquence (cohérence horizontale). Ces centres de décision seront amenés à se coordonner de manière à assurer, à leur niveau de vision, l'ensemble des fonctions d'une chaîne logistique.

Nous proposons parallèlement de construire avec le langage SADT, la vue des processus opérationnels (système physique) agrégée et partagée par les centres de décision d'un même niveau (cf. flèche 1 de la Figure 6). De ce fait, chaque centre de décision pourra définir quelles sont les activités qu'il pilote (cf. flèches 2 de la Figure 6). Néanmoins, plusieurs centres de décision d'un même horizon/période pourront gérer complémentairement une même activité. Par exemple, l'un fixera les consignes et l'autre fixera les moyens.

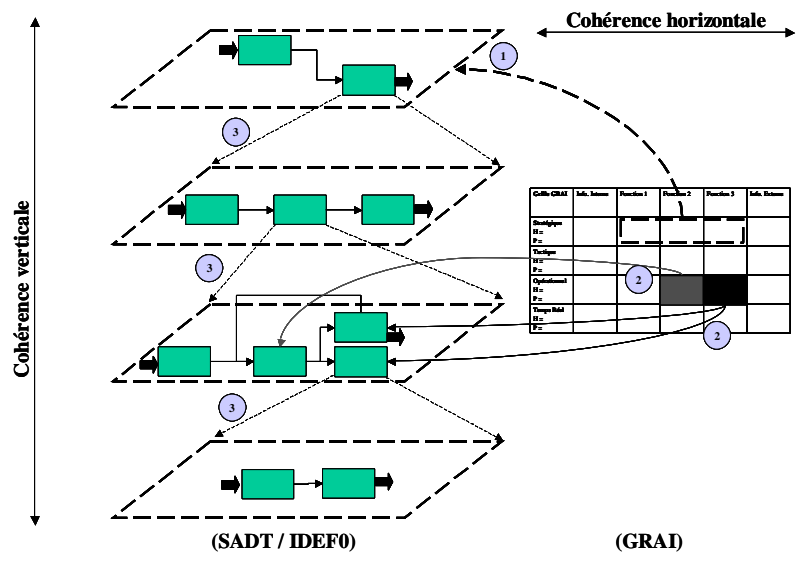

Figure 7. Cohérence des processus opérationnels et de pilotage. 
Verticalement, apparaissent dans une colonne de la grille GRAI des centres de décision qui pilotent une même fonction à des horizons différents. La décomposition en activités des processus opérationnels vus d'un niveau de décision peut donc définir la vue fonctionnelle du niveau de décision inférieur. Ceci garantit que tout processus opérationnel est identifié dans un et un seul processus du niveau supérieur. On parlera alors de cohérence verticale.

La décomposition hiérarchique des processus telle que le permet la méthode SADT rend possible un raffinement progressif et cohérent des processus (cf. flèches 3 de la Figure 6). L'idée de calquer les différents niveaux de cette décomposition sur les lignes de la grille GRAI fournit de la cohérence.

Par construction, cette vue fonctionnelle présente plusieurs propriétés utiles à la formulation des indicateurs de performance :

- elle fournit les éléments (consignes, moyens et résultats) utilisés et leurs unités à chaque niveau de décision ;

- elle indique comment l'agrégation des activités d'un niveau pourra constituer la vue de niveau supérieur. Par suite, elle aide à formuler, par ce mécanisme d'agrégation, les éléments (consignes, moyens et résultats) d'un niveau à partir du niveau inférieur.

\subsection{Processus de conception du système d'indicateurs de performance}

Notre méthodologie est constituée de six phases.

Phase 1 : On représente le système de pilotage sur la base de grilles GRAI . Dans le cadre d'une organisation distribuée, plusieurs grilles GRAI sont nécessaires (une par entité indépendante) et doivent être connectées pour faire apparaître, à un niveau macroscopique, la propagation des cadres de décision, et donc des objectifs, dans l'ensemble du système (déploiement de la stratégie). La construction des grilles permet d'identifier les déterminants de la performance de chacun des centres, selon la méthode ECOGRAI [Bitton, 1990] : les objectifs d'un centre de décision sont obtenus par décomposition de ceux de la fonction à laquelle il appartient; les déterminants sont les variables du décideur permettant d'atteindre les objectifs. En pratique, les déterminants sont extraits d'interviews auprès des acteurs de chaque centre de décision et validés par ces mêmes décideurs.

Phase 2 : Cette phase modélise les processus opérationnels (physiques) vus par chaque horizon décisionnel (i.e. ligne d'une grille GRAI élaborée en phase 1). Des modèles SADT caractérisent les processus réalisés, les ressources et entrées utilisées, les consignes et les sorties produites tout en respectant les règles d'articulation définies au paragraphe 4.2. Là encore ce sont des entretiens auprès des acteurs qui vont permettre d'établir et de valider le modèle de processus. 
Phase 3 : On projette les variables de décision sur les modèles d'activités qui composent les processus opérationnels. On peut, dès lors, substituer les noms d'activités par ceux de ces déterminants de performance. Cette étape met en évidence, pour chaque activité, les éléments de référence utiles à la définition des indicateurs de performance sur les points de vue. Pour la mise en œuvre, le responsable de chaque centre de décision validera la propagation de ses déterminants sur les activités unitaires des processus opérationnels dont il a la charge.

Phase 4 : Pour chaque niveau de décision et pour chaque activité, les utilisateurs formulent des indicateurs de performance. Les unités temporelles et la période de validité d'un indicateur correspondent à la période et à l'horizon du niveau de décision. Les unités de flux ou de moyens sont fixées par le modèle SADT. L'utilisation de formulations standard issues d'experts de terrain ou de bonnes pratiques (SCOR, [Eymery, 1997] ou [Gunasekaran et al., 2004]) est préconisée.

Phase 5 : Les différents tableaux de bord sont implémentés dans une structure respectant les points de vue, et dans une optique de contrôle et de régulation.

Phase 6 : On peut utiliser les portefeuilles d'indicateurs constitués pour conduire des actions d'amélioration continue de la performance du système logistique. Ceci peut conduire à redéfinir: les responsabilités (prises de décision), les processus opérationnels ou les leviers d'action/décision. Les modèles GRAI ou SADT pourront alors être mis à jour. Ce qui amène à boucler sur les phases 1 ou 2 .

Dans la suite, nous ne développons que les phases 1 à 4 . Les phases 5 et 6 étant relatives à l'implémentation et à l'exploitation du système d'IP.

\subsection{Etude des propriétés de la méthode}

La méthode préserve certaines des propriétés que possèdent les méthodes desquelles elle s'est inspirée (cf. Tableau 1):

- Elle permet d'associer les indicateurs de performance aux activités qui composent les processus du système étudié. Cette propriété découle de la construction proposée du système d'indicateurs de performance à partir d'un modèle de processus (SADT).

- Elle offre la capacité de définir des indicateurs de performance relativement aux déterminants de la performance. Cette propriété est la conséquence de l'identification des déterminants par le modèle de représentation décisionnel et de leur propagation dans le modèle de processus.

- Elle lie les indicateurs de performance aux centres de décision qui les utiliseront, et garantit la dualité de ces indicateurs vis-à-vis des processus de pilotage et des processus opérationnels. Cette propriété est issue de la construction des indicateurs sur la base d'une cartographie croisée des processus décisionnels et opérationnels. 
- Elle permet l'agrégation des indicateurs d'activités d'une même fonction : possibilité d'agréger les résultats sur les différents niveaux de décision d'une même fonction ${ }^{6}$.

- Elle assure une cohérence temporelle dans la définition des indicateurs de performance des centres de décision d'un niveau de décision. Cette propriété est directement sous-jacente à la structuration même de la grille GRAI.

A ces premières caractéristiques viennent s'ajouter des avantages pratiques liés à notre proposition d'introduire les « points de vue ambition, réalité et aisance » :

- Il s'agit de la localisation des origines de la (non-)performance. Cette propriété est due au principe même de définition des points de vue. En effet, ils permettent de mesurer la sensibilité des déterminants sur les moyens, consignes et résultats. Ainsi, il est possible de situer une défaillance sur ces axes d'analyse et de situer plus précisément les origines du dysfonctionnement.

- Un corollaire immédiat est l'identification d'axes de progrès (puisque la méthode permet de disposer d'un système d'IP qui indique précisément les sources de (non-)performance) pour résorber les dysfonctionnements constatés, ou pour consolider les organisations performantes.

\begin{tabular}{|c|c|c|c|c|c|}
\hline $\begin{array}{c}\text { Propriétés des modèles de } \\
\text { définition de systèmes } \\
\text { d'indicateurs de performance }\end{array}$ & \multicolumn{5}{|c|}{$\begin{array}{c}\text { Existence de la propriété } \\
\text { dans chaque méthode }\end{array}$} \\
\hline $\begin{array}{c}\text { Notre } \\
\text { méthode }\end{array}$ & ABC/M & $\begin{array}{c}\text { ECO- } \\
\text { GRAI }\end{array}$ & BSC & SCOR \\
\hline $\begin{array}{c}\text { Association Indicateur } \\
- \text { Activités }\end{array}$ & ++ & ++ & - & + & ++ \\
\hline $\begin{array}{c}\text { Indicateur fonction } \\
\text { Déterminants }\end{array}$ & ++ & - & ++ & ++ & + \\
\hline $\begin{array}{c}\text { Association Indicateur } \\
- \text { Décision }\end{array}$ & + & + & ++ & + & + \\
\hline $\begin{array}{c}\text { Cohésion temporelle } \\
\text { Indicateurs }\end{array}$ & + & - & ++ & - & - \\
\hline $\begin{array}{c}\text { Cohérence fonctionnelle } \\
\text { Indicateurs }\end{array}$ & ++ & + & + & + & + \\
\hline $\begin{array}{c}\text { Identification directe } \\
\text { des causes }\end{array}$ & ++ & - & + & - & - \\
\hline $\begin{array}{c}\text { Facilitation diagnostic } \\
\text { élaboration plan actions }\end{array}$ & ++ & + & + & + & + \\
\hline \multicolumn{2}{|l|}{ Légende : ++ totalement $;+=$ partiellement $;-=$ pas du tout } \\
\hline
\end{tabular}

Tableau 1. comparaison de la méthode proposée avec d'autres méthodes.

${ }^{6}$ Notons ici que l'agrégation suppose que l'on travaille sur des données brutes en valeur, et jamais sur des ratios comme le préconise la définition des points de vue. 


\section{Illustration par l'exemple}

L'exemple est extrait d'une mission réalisée par les auteurs dans une grande entreprise fabriquant des produits pharmaceutiques et cosmétiques en France, et distribuant vers un marché international en pleine croissance. Cette croissance s'accompagne de la création et du développement de filiales dans les différents pays où les marchés sont porteurs. La question posée est de piloter l'évolution de la logistique intra-entreprise dans un contexte d'internationalisation rapide des activités.

La méthode a été utilisée pour évaluer et aider au pilotage de cette chaîne logistique, ${ }^{7}$ et faire évoluer les modes de fonctionnement de la coopération entre la maison-mère qui assure l'essentiel de la production des articles, et les filiales étrangères qui assument la relation aux clients (gestion de la demande, stockage, distribution, vente, SAV).

Nous illustrons ici quelques résultats obtenus au cours de l'application des 4 premières phases de la méthode proposée.

\subsection{Phase 1 : Définition du modèle décisionnel}

La décomposition temporelle nous a conduit à considérer six niveaux décisionnels :

- Politique (Horizon 10 ans, Période 1 an) portant sur les nouveaux marchés, l'actionnariat, la R\&D ou encore les partenariats.

- Schémas Directeurs (Horizon 5 ans, Période 1 an) portant sur les organisations, les investissements à long terme, les schémas de production, de distribution.

- Stratégique (Horizon 18 mois, Période 1 mois) portant sur les investissements à moyen terme, les développements produits, la planification et l'arbitrage capacitaire relatif aux familles de produits.

- Tactique (Horizon 12 mois, Période 1 mois) portant sur les investissements à court terme, les développements des supports à la vente, la planification et l'arbitrage capacitaire relatifs aux produits.

- Opérationnel (Horizon 1 mois, Période 1 semaine) portant sur l'ajustement des ressources, l'ordonnancement et la gestion de la relation clients.

\footnotetext{
${ }^{7}$ Il faut remarquer ici la caractéristique de cette application industrielle qui est une chaîne logistique interne. Ainsi, il est peut-être plus difficile dans le cas de chaînes logistiques inter-entreprises de mettre en œuvre ce genre d'approche : problème accru de transparence des informations et plus globalement de confiance. Néanmoins, chaque filiale du cas étudié étant parfaitement autonome dans la gestion de ses processus opérationnels, l'application reste représentative.
} 
- Temps réel (Horizon 1 semaine, Période temps réel) portant sur le traitement des commandes, la réalisation et l'expédition des produits.

La grille GRAI de la Figure 8 fait apparaître les niveaux de coopération entre la maison-mère et ses filiales en représentant les relations entre les centres de décision des 2 types d'entités. Il apparaît une relation unilatérale de cadres définis par la maison-mère et «imposés » aux filiales sur les niveaux «Politique et Schémas Directeurs ». Ceci démontre une volonté de laisser une autonomie au niveau de chaque filiale pour la définition propre de ces cadres de décision, dès le niveau «Schémas Directeurs ».

\begin{tabular}{|c|c|c|c|c|c|c|c|c|c|c|c|c|}
\hline & \multicolumn{6}{|c|}{ Fonctions Maison Mere } & \multicolumn{6}{|c|}{ Fonctions Filiales } \\
\hline Horizon & Info. Ext. & Concevoir & Gérer Client & Gérer Prod. & \begin{tabular}{|l|} 
Gérer Dist. \\
\end{tabular} & Info. Int. & Info. Ext. & Concevoir & Gérer Client & Gérer Prod. & \begin{tabular}{|l|} 
Gérer Dist. \\
\end{tabular} & Into. Int. \\
\hline POLITIQUE & & & & & 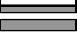 & & & 5 & & & & \\
\hline$H=10$ ans & & & & & & & & & - & & & \\
\hline$P=1$ an & & & & & 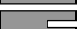 & & & & & & $=$ & \\
\hline SCHEMAS & & & & & ए & & & $E$ & & & & \\
\hline DIRECTEURS & & & & & 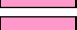 & & & 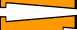 & & & & \\
\hline $\begin{array}{l}=5 \text { ans } \\
P=1 \text { an }\end{array}$ & & & & & $\overline{7}$ & & & & & & - & \\
\hline $\begin{array}{c}\text { STRATEGIQUE } \\
H=18 \text { mois } \\
P=1 \text { mois }\end{array}$ & & $\begin{array}{c}\text { Développer } \\
\text { Produits }\end{array}$ & $\begin{array}{l}\text { Prévoir } \\
\text { Familles }\end{array}$ & $\begin{array}{l}\text { Réaliser } \\
\text { PIC } \leftarrow\end{array}$ & $\begin{array}{l}\text { Planifier } \\
\text { Charges }\end{array}$ & Historique & & $\begin{array}{c}\text { Développer } \\
\text { Produits }\end{array}$ & $\begin{array}{l}\text { Prévoir } \\
\text { Eamiles }\end{array}$ & \begin{tabular}{|l} 
Réaliser \\
- PIC
\end{tabular} & $\begin{array}{l}\text { Planifier } \\
\text { Charges }\end{array}$ & Historique \\
\hline $\begin{array}{l}\text { TACTIQUE } \\
H=12 \text { mois } \\
P=1 \text { mois }\end{array}$ & & $\begin{array}{c}\text { Développer } \\
\text { Supports }\end{array}$ & 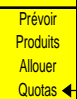 & $\begin{array}{l}\text { Réaliser PDP } \\
\text { et CBN } \\
\text { (MRP) } \\
\end{array}$ & $\begin{array}{l}\text { Planifier } \\
\text { Besoins } \\
\text { Canacité }\end{array}$ & Historique & & $\begin{array}{c}\text { Développer } \\
\text { Supports }\end{array}$ & $\begin{array}{l}\text { Prévoir } \\
\text { Produits } \\
\text { Allouer } \\
\text { Yuotas }\end{array}$ & $\begin{array}{c}\text { Réaliser PDP } \\
\text { et CBN } \\
\text { (DRP) }\end{array}$ & $\begin{array}{l}\text { Planifier } \\
\text { Besoins } \\
\text { Capacité }\end{array}$ & Historique \\
\hline $\begin{array}{l}\text { OPERATIONNEL } \\
\qquad \begin{array}{l}H=1 \text { mois } \\
P=1 \text { sem. }\end{array}\end{array}$ & & & $\begin{array}{l}\text { Gérer } \\
\text { Comptes } \\
\text { Clients }\end{array}$ & Appro. 4 & $\begin{array}{l}\text { ordonance } \\
\text { livraisons }\end{array}$ & & & & $\begin{array}{c}\text { Gérer } \\
\text { Comptes } \\
\text { Clients }\end{array}$ & $\begin{array}{l}\text { Appro. } \\
\text { Ordonnancer }\end{array}$ & $\begin{array}{c}\text { Ordonnancer } \\
\text { livraisons }\end{array}$ & \\
\hline $\begin{array}{c}\text { TEMPS REEL } \\
H=1 \text { sem. } \\
P=\text { temps réel }\end{array}$ & & & $\begin{array}{c}\text { Enregistrer } \\
\text { Commandes } \\
\text { Facturer } \\
\text { Filiales }\end{array}$ & $\begin{array}{l}\text { Fabriquer } \\
\text { Produits }\end{array}$ & $\begin{array}{l}\text { Réception. } \\
\text { Stocker } \\
\text { Préparer } \\
\text { Expédier }\end{array}$ & & & & $\begin{array}{c}\text { Enregistrer } \\
\text { Commandes } \\
\text { Facturer } \\
\rightarrow \text { Filiales }\end{array}$ & $\begin{array}{c}\text { Fabriquer } \\
\text { Produits }\end{array}$ & $\begin{array}{l}\text { Réception. } \\
\vec{P}_{\text {Stocker }} \\
\text { Préparer } \\
\text { Exoédier }\end{array}$ & \\
\hline
\end{tabular}

Figure 8. relation entre les grilles GRAI maison-mère et filiale.

Au niveau « Politique », chaque centre de décision a pour objet la définition de la stratégie relative à sa fonction (Définir la stratégie de production, pour la fonction Gérer la production par exemple). Au niveau "Schémas Directeurs », chaque centre de décision a pour objet la définition des moyens et des modalités de réalisation (processus) nécessaires pour mettre en œuvre la stratégie. Il s'agit là des 2 classes de déterminants de la performance associés à chaque centre de décision du niveau « Schémas Directeurs ».

\subsection{Phase 2 : Identification et formalisation des processus}

La Figure 9 présente, pour le niveau «Schémas Directeurs », la vision du système logistique en termes de processus opérationnels. A ce niveau, la perception des processus par les décideurs se limite à une activité élémentaire par fonction. 


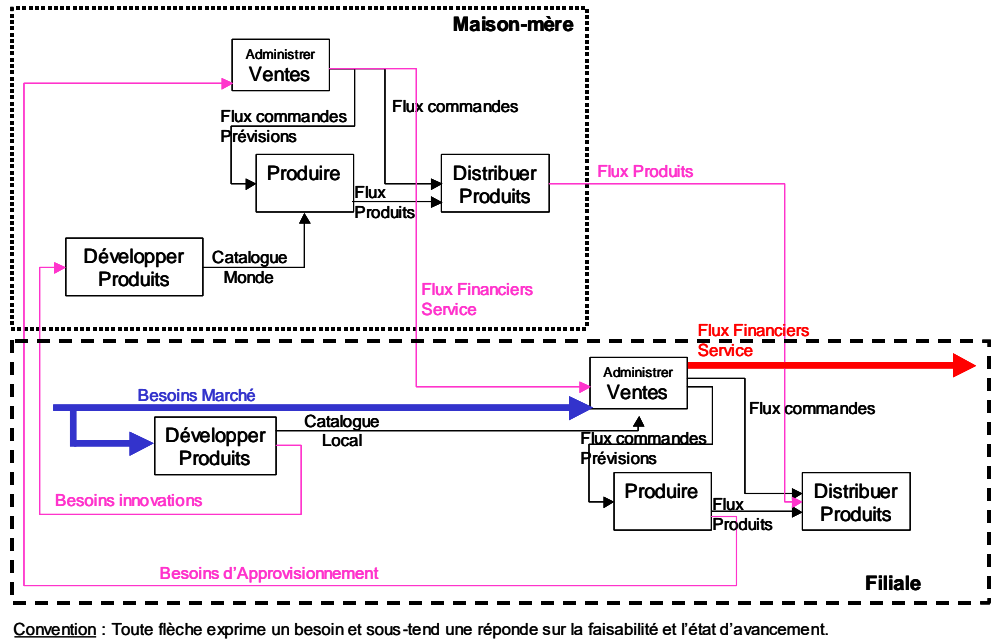

Figure 9. Vision de l'activité au niveau « Schémas Directeurs ».

La Figure 10 développe la vision «Stratégique » de l'activité «Produire » de la maison-mère. Les objets manipulés sont des familles de produits et des macroressources. Par construction (hiérarchisation et décomposition SADT), les entrées (prévisions et commandes), les sorties (produits et services), les consignes (services et coûts) et les ressources (Usines, compétences et outils) indiquées sur le modèle «Schémas Directeurs » se retrouvent sur le niveau de modélisation « Stratégique ».

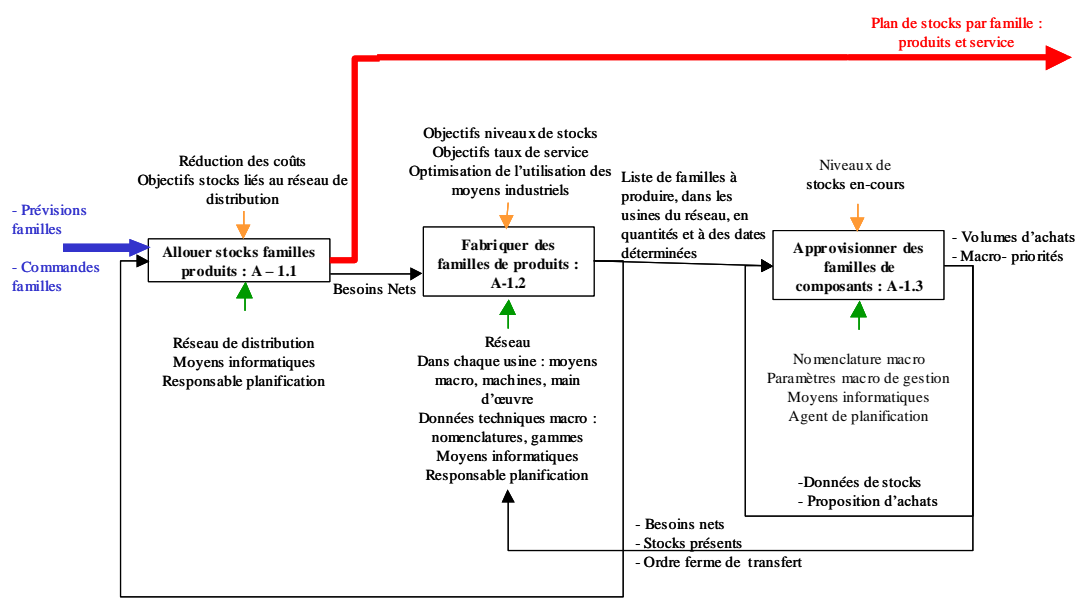

Figure 10. Activité « Produire » : Niveau Décisionnel « Stratégique ». 


\subsection{Phases 3 et 4 : Définition du système d'évaluation de performance}

Les responsables pour l'horizon décisionnel «Schémas Directeurs» de la fonction « Produire » ont pu préciser la nature de leurs déterminants :

- Adapter les ressources (humaines, financières et matérielles) : décider de l'ouverture / fermeture d'une usine de production ou ligne de fabrication, décider d'un plan de formation et/ou recrutement sur des compétences particulières, contractualiser un contrat de service avec un sous-traitant, etc. ;

- Paramétrer les processus de planification et d'exécution: réviser les procédures internes de planification, concevoir un nouveau mode de dimensionnement des stocks, décider d'une règle d'arbitrage et de gestion des priorités en cas de pénurie, etc.

Dès lors, les indicateurs de performance et les points de vue ont été formulés par ces mêmes experts métiers sur la base des indicateurs déjà existants dans l'entreprise (validés et contextualisés grâce à la démarche suivie), de bonnes pratiques (modèle SCOR essentiellement) et de propositions complémentaires des décideurs.

La Figure suivante illustre le résultat de cette démarche. Le nom de l'activité réalisée a été éliminé de la représentation au profit des déterminants de la performance. Cette activité dispose de consignes de coûts à minimiser et de niveau de service à maximiser (respect des engagements clients, en l'occurrence, mise à disposition des produits pour la vente). Ces consignes sont cohérentes avec les sorties de l'activité que sont les produits fabriqués et le service associé. Les ressources sont de type :

- matérielles : usines de production, lignes de fabrication (réacteurs et des lignes de conditionnement), produits composants ;

- humaines : compétences individuelles et collectives, organisation (nombre d'équipes, intérimaires...) ;

- supports : systèmes d'information et outils techniques (contrôle qualité...).

L'utilisation des ressources est contrainte par les moyens financiers disponibles et alloués à cette activité (tant sur le plan du fonctionnement que des investissements). Les indicateurs de performance et les points de vue qui ont été définis avec les experts métiers de la chaîne logistique sont les suivants :

- indicateurs de performance des résultats : le nombre d'unités produites (par site) et le taux de rupture (nombre de commandes, par site, non livrées dues à un stock nul) de façon à permettre le suivi, respectivement, des flux de produits et de la qualité de service rendue ;

- $\quad$ indicateurs de suivi des déterminants : le coût relatif de l'activité, les niveaux de couvertures de stock (nombre de mois de consommation prévisionnelle présente en stock) des produits finis ( $\mathrm{PF}$ ) et des composants ( $\mathrm{Cp}$ ), ainsi que le temps de cycle. Ces indicateurs permettent de suivre conjointement 
l'adaptation des ressources (coûts) et l'optimisation des processus (couvertures et temps de cycle);

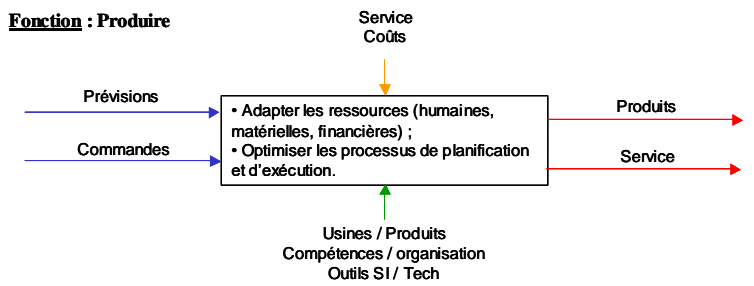

\begin{tabular}{|c|c|c|c|c|}
\hline \multicolumn{2}{|c|}{ Indicateurs de Performance } & \multicolumn{3}{|c|}{ Points de vue } \\
\hline Suivi des Résultats & Suivi des Déterminants & PV Ambition & PV Réalité & PV Aisance \\
\hline Nom & Nom & Nom & Nom & Nom \\
\hline Unités & Coût relatif de l'activité & Déviation coût relatif & Productivité & Taux de ss-traitance \\
\hline Taux de rupture & Couverture de stocks $\mathrm{Cp}$ & Déviation Couverture & Taux d'utilisation & Taux Intérimaires \\
\hline & Couverture de stocks PF & Déviation Couverture & Taux d'obsolescence & Taux de panne \\
\hline & Temps de cycle & Déviation Temps Cycle & Taux de rebuts & Taux Non Qualité \\
\hline
\end{tabular}

Figure 11. Exemple de structuration de tableau de bord.

- points de vue ambition (lien entre déterminants et consignes) : il s'agit de mesurer l'impact des déterminants « adapter les ressources » et «paramétrer les processus » sur les consignes de coûts et de services. Les indicateurs de ce point de vue mesurent les écarts relatifs (déviation) entre les consignes de coût allouées à cette activité et la réalité des coûts (exprimés en termes numéraires et en termes de couvertures de stocks, cf. indicateurs de suivi des déterminants) engagés par les déterminants de cette activité. De la même façon, les indicateurs de ce point de vue mesurent les écarts relatifs en termes de service (temps de cycle de l'activité) ;

- points de vue réalité (lien entre déterminants et résultats) : suivi des critères de productivité (unités produites / jour), de taux de service (commandes complètes livrées à dates), de niveau d'obsolescence (unités obsolètes / total unités) et de taux de retour (unités retournées / total unités). Ces quatre critères rapportent bien à l'usage fait des leviers «adaptabilité des ressources » (utilisation, obsolescence) et "paramétrage des processus » (productivité, rebuts) pour fabriquer les produits et rendre le service aux clients (sorties) ;

- point de vue aisance (lien entre déterminants et ressources) : suivi du problème de dimensionnement par les déterminants des ressources pour la bonne activation des activités notamment en identifiant l'utilisation faite de moyens humains et métiers externes (taux d'intérimaires, taux de soustraitance). En ce qui concerne les ressources techniques et matérielles, un suivi de l'impact de leur usage sur les processus par l'évaluation des taux de non qualité composants et d'utilisation des machines (heures effectivement utilisées / heures disponibles) a été retenu. 
La définition des indicateurs ne résulte pas d'une identification précise des liens de causalité entre variables prises deux à deux. Il s'agit davantage, par exemple pour le point de vue réalité, d'une expression "générale » de la sensibilité des relations entre l'ensemble des déterminants et l'ensemble des sorties.

L'opération a bien entendu été répétée sur la totalité des activités identifiées par la modélisation fonctionnelle. Ce sont, pour les seuls niveaux décisionnels « Politique » et «Schémas Directeurs », près de 30 indicateurs de performance qui ont été définis et près de 50 points de vue (pour partie déjà existants dans l'entreprise, mais pas exploité de façon ciblée). Les indicateurs et points de vue définis ont tous été associés aux déterminants de la performance des activités étudiées. Rappelons que les points de vue ont vocation à être analysés pour préciser les causes d'un dysfonctionnement sur un ou plusieurs de ses indicateurs.

\subsection{Conclusion}

Nous avons montré comment définir un système d'indicateurs de performance pour le pilotage de processus en fonction de ses déterminants. Nous avons illustré l'intérêt d'une telle approche sur le cas d'une chaîne logistique intra-entreprise.

La méthodologie que nous avons proposée reprend les bonnes propriétés avancées par les principales méthodes existantes de définition de systèmes d'indicateurs de performance, et tente de les compléter en offrant notamment un moyen d'identifier de façon ciblée, les causes d'une (non-) performance à l'aide des «points de vue » efficience et efficacité et pertinence.

Nous identifions plusieurs axes de réflexion complémentaires à la suite de ces travaux.

D'abord, l'appropriation du modèle par l'industriel, même si elle est en cours ${ }^{8}$, n'est pas totalement validée. Si les responsables de la chaîne logistique étudiée ont aujourd'hui trouvé dans cette approche un outil qui permettra de mieux piloter le système, il reste à s'interroger sur la réelle capacité de notre proposition à s'adapter aux évolutions très rapides de contexte et d'organisation.

Ensuite, la mise en œuvre d'une telle démarche suppose, au niveau de la gestion de la chaîne logistique, d'intégrer de façon plus formelle les actions de pilotage par la performance. Cette dimension pose encore trop souvent des difficultés de mise en œuvre pour définir des outils adaptés et les exploiter, notamment sur les questions de coordination qui renvoient à des pouvoirs et des responsabilités. Une telle évolution peut donc engendrer des changements importants et, le cas échéant, bouleverser les

${ }^{8}$ L'entreprise met en œuvre la démarche pour redéfinir l'ensemble de ses tableaux de bord logistique de l'horizon décisionnel « Politique » à «Temps réel ». La quasitotalité des indicateurs est aujourd'hui définie. Leur implémentation est en cours. 
comportements et les organisations. Ce sont ces changements et leurs impacts qu'il conviendrait de considérer plus avant.

Par ailleurs, notre proposition génère mécaniquement une masse importante d'information (nombre d'indicateurs et points de vue). Une réflexion sur la viabilité de la gestion d'une telle masse d'information doit être conduite. Nous avons commencé à travailler à la construction d'un cube de la performance offrant plusieurs niveaux d'analyse et d'exploitation (agrégation, multi-critères) pour faciliter la prise de décision [Lauras et al., 2006].

En outre, notre étude a traité le cas d'une chaîne logistique intra-entreprise. Pourtant, nous l'avons vu, la chaîne logistique doit intégrer l'ensemble des acteurs depuis le fournisseur $\mathrm{du}$ fournisseur au client $\mathrm{du}$ client. Par ailleurs, l'expérimentation a été intégralement conduite sur le cas d'une chaîne logistique pharmaceutique et cosmétique. Il est sans doute judicieux d'étudier la faisabilité d'étendre ce type de solutions aux partenaires industriels, qu'ils soient en amont ou en aval de l'entreprise. Existe-t-il des limites à l'extension, de quelle nature ? On peut également s'interroger sur les capacités de transposition de nos résultats à d'autres types de chaînes logistiques disposant d'autres catégories de contraintes.

Enfin, nous avons émis, au début de cette étude, une hypothèse majeure précisant que les objectifs, organisations et fonctions du système étudié était connus et figés. Il apparaît à présent opportun d'envisager des extensions aux solutions proposées, intégrant la possibilité de (re)concevoir tout ou partie du système, tant en termes d'organisation que de définition de processus. Une telle perspective suppose des évolutions sensibles sur la boucle de pilotage et donc, sur l'évaluation de performance sous-jacente.

\section{Bibliographie}

AFNOR, Normes Qualité ISO 9000, Editions AFNOR, 2000

Al-Mudimigh, A.S, Zairi M., Ahmed A.M.M., Extending the concept of supply chain: the effective management of value chains, International Journal of Production Economics, No $87,309-320,2004$

Amaratunga, D., Baldry D., Moving from performance measurment to performance management, Facilities, No 20, 5-6, 2002

Beamon, B.M., Measuring Supply Chain Performance. International Journal of Operations and Production Management, Vol. 19, No 3, 275-292, 1999

Berrah L., Haurat A., Une stratégie de mise en place d'indicateurs de performance pour le pilotage de processus de production, MOSIM, Rouen, 1997

Berrah L., Mauris G., Foulloy L., Haurat A., Global vision and performance indicators for an industrial improvement approach, Computers in Industry, $\mathrm{N}^{\circ}$ 43, p. 221-225, 2000

Bescos P.L., Dobler P., Mendoza C., Naulleau G., Contrôle de gestion et management, Editions Montchrestien, Guide des techniques et de la décision, Paris, 1995 
Bitton M., ECOGRAI : méthode de conception et d'implantation de systèmes de mesure de performance pour organisations industrielles, Thèse de doctorat, Université Bordeaux I, septembre 1990

Bolstorff P., How does SCOR measure up ? Supply Chain Technology news, http://www.totalsupplychain.com, p. 22-25, 2002

Bourguigon A., Sous les pavés la plage... ou les multiples fonctions du vocabulaire comptable : l'exemple de la performance, Comptabilité - contrôle - audit, p. 89 à 101, mars 1997

Burlaud A., A la recherche d'un système de mesure des performances : application aux réseaux, CEFAG, février 1999

Burlat P., Modélisation et pilotage des organisations en réseau, Habilitation à Diriger les Recherches, Ecoles des Mines de Saint-Etienne, 2002

Burlat P., Marcon E., Sénéchal O., Dupas R., Berrah L., Concepts pour la performance des systèmes de production, Chapitre d'ouvrage «Evaluation des performances des systèmes de production » sous la direction de C. Tahon, Edition Hermès, p.49-80, 2003.

Burlat P., X. Boucher, Une utilisation de la théorie des sous-ensembles flous pour le calcul d'indicateurs de performance, MOSIM, Toulouse, 2003

Chow, G., Heaver T.D., Henriksson L.E., Logistics performance: Definition and measurement, International Journal of Physical Distribution \& Logistics Management, No 24, 17-28, 1994

Cokins, G., Activity-based Cost Management: an Executive's Guide, Edition hardcover, 2001

Debauche B., Mégard P., Business Process Management : pilotage métier de l'entreprise, Editions Hermès, Collection Lavoisier, 212 p., 2004

Doumeingts G., Vallespir B., Modélisation d'entreprise : vers le système d'information, Actes de l'école de modélisation d'entreprise, Ecole des Mines d'Alès, 2004

Doumeingts G., La méthode GRAI, Thèse d'Etat, Université Bordeaux I, 1984

Ducq, Y., Vallespir B. and Doumeingts G., Coherence analysis methods for production systems by performance aggregation, International Journal of Production Economics, No $69,23-37,2001$

Ducq Y., Gentil M.H., Merle C., Doumeingts G., Conception et implantation de systèmes d'indicateurs de performance, Evaluation des performances des systèmes de production, C. Tahon Edt, Edition Hermès, p.143-174, 2003

Eymery P., La logistique de l'entreprise : Supply Chain Management, Edition Hermes Systèmes d'information, 217 p., 1997

Folan, P., Browne J., A review of performance measurement: towards performance management, Computers in Industry, Article in Press, 2005

Forrester, J.W., Principles of Systems, Cambridge, Mass: Wright-Allen Press, 1968

Frein Y., Evaluation de performances pour la conception des flux, Université d'été du pôle productique Rhône-Alpes, 1998

Giard V., Gestion de la production et des flux, Editions Economica, 2003

Gray, C.D., Does your supply chain system measure up?, APICS - The Performance Advantage, January, 1999 
Gunasekaran A., Patel C., Ronald E., McGaughey E., A framework for supply chain performance measurement, International Journal of Production Economics, 2003

Hammer M., Champy J., Le re-engineering : réinventer l'entreprise pour une amélioration spectaculaire de ses performances, Editions Dunod, 1993

Jacot J.-H., A propos de l'évaluation économique des systèmes intégrés de production, ECOSIP - Gestion industrielle et mesure économique, Economica, 1990.

Kaplan R.S., Norton D.P., Le tableau de bord prospectif, traduit de l'américain "The Balanced Scorecard », Editions d'Organisation, 311 p., 1996

Kaplan R.S., Norton D.P., Comment utiliser le tableau de bord prospectif, Les éditions d'organisation, 440 p., 2001

Lauras M., Méthodes de diagnostic et d'évaluation de performance pour la gestion de chaînes logistiques, Thèse du Centre de Génie Industriel de l'Ecole des Mines d'AlbiCarmaux, INP Toulouse n²119, 7 Juillet 2004

Lauras, M., Pingaud H., Lamothe J., Management of supply chain processes with the REED method, INCOM - IFAC, France, 2006

Lee, H.L., Creating Value through supply chain integration, Supply Chain Management Review, 2000

Lissandre M., Maîtriser SADT, Editions Armand Colin, 1990

Lohman F.A.B., The effectiveness of management information, Ph.D. Thesis, Delft University, 1999

Lohman, C., Fortuin L., Wouters M., Designing a performance measurement system: a case study, European Journal of Operation Research, No 156, 267 - 286, 2004

Mélèze, J., Approches systémiques des organisations, Ed. d'Organisation, 1990

Morley C., La modélisation des processus : typologie et proposition utilisant UML, Assises ADELI, 2002

Neely, A.D., Gregory M., Platts K., Performance measurement system design, International Journal of Operations \& Production Management, No 15, 80 - 116, 1995

Ravignon L., Bescos P.-L., Joalland M., Le Bourgeois S., Malejac A., La méthode ABC/ABM : piloter efficacement une PME, Editions d'Organisation, 1998

Roboam M., La méthode GRAI : principes, outils, démarche et pratique, Edition TEKNEA, 226 p., 1993

Smith, P., Goddard M., Performance management and operational research: a marriage made in heaven?, Journal of the Operational Research Society, No 53, 247 - 255, 2002

Stadtler H., Kilger C., Supply Chain Management and Advanced Planning, Edition SpringerVerlag, 371 p., 2000

Supply Chain Council, Supply Chain Operations reference-models - SCOR version 6.0, Edition Supply Chain Council, 2004

Tahon C., Frein Y., Document de synthèse du Groupe de Recherches en Productique Thème 4 : évaluation de performances, 1999

Vernadat F., Techniques de modélisation en entreprise : application aux processus opérationnels, Editions Economica, 1999 